\title{
Article
}

\section{Origin and Position of Cystic Artery in Bangladeshi Corpse}

\author{
Khalil M ${ }^{1}$, Sultana $\mathrm{ZR}^{2}$, Rahman $\mathrm{HR}^{3}$, Sultana $\mathrm{SZ}^{4}$, Mannan S ${ }^{5}$, Rahman MM ${ }^{6}$, Ahamed \\ MS ${ }^{7}$, Chowdhury MAS ${ }^{8}$
}

This cross sectional descriptive morphological study was done to see the site of origin of cystic artery supplying the gallbladder in Bangladeshi people to increase the knowledge regarding variation anatomy in our population. Sixty post mortem tissue blocks containing liver and gallbladder along with surrounding structures were collected from 40 male and 20 female cadavers of different age groups and fixed in 10\% formal saline solution. This study was carried out in the Department of Anatomy in Mymensingh Medical College, Mymensingh , from July 2007 to June 2008. Gross and fine dissections were carried out to study the different origin of cystic artery and its topographic relationship with adjacent structures. In the present study, findings were compared with the findings of Western researchers. In the present study, the so-called typical origin of the cystic artery from right hepatic artery was $90 \%$ and in $10 \%$ cases it was found to arise from other sources. Out of $10 \%, 3 \%$ arise from left hepatic artery, $3 \%$ from junction between right and left hepatic artery, $2 \%$ from hepatic artery proper and $2 \%$ from gastro duodenal artery.

Key Wards: Cystic artery, Origin, Location.

J Bangladesh Soc Physiol.2008 Dec;(3):66-70. For author affiliations, see end of text.

http://www.banglajol.info/index.php/JBSP

\section{Introduction}

$\mathbf{L}$ Liver and billiary diseases are the most common health problem throughout the world as well as in Bangladesh. Gross morphology and histology of the liver and extra hepatic billiary apparatus are the most important parameter to know the disease process as well as for treatment purpose, especially during surgical intervention. Cholecystitis, cholelithiasis, choledocholithiasis are the most common biliary problem. People with severe biliary colic - intense spasmodic pain in the right upper quadrant of the abdomen resulting from impaction of a gallstone in the cystic duct and acute inflammation of the gallbladder (cholecystitis) - may have their gallbladders removed. Laparoscopic cholecystectomy often replaces the open surgical method. Dissection of the cystohepatic triangle (Calot's triangle) - bounded by the cystic artery, cystic duct and common hepatic duct - early during cholecystectomy safeguards these important structures should there be anatomical variations. Errors during gallbladder surgery commonly result from failure to appreciate the common variations in the anatomy of the biliary system, especially its blood supply. Before dividing any structure and removing the gallbladder, surgeons identify all three biliary ducts, as well as the cystic and hepatic arteries. It is usually the right hepatic artery that is in danger during surgery and observation of its exact location is essential before ligating the cystic artery ${ }^{1}$.

A study done on nineteen operated patients for choledochal cyst over a 7- years period. About one-third of those patients (6 out of 19) had biliary

J Bangladesh Soc Physiol. 2008 Dec;(3):66-70 
ductal / vascular anomalies identified at surgery 2. A high degree of awareness of biliary ductal and vascular anomalies is required on the part of the operating surgeon to facilitate accurate intraoperative diagnosis as well as successful reconstructive surgery.

The cystic artery is usually a branch of the right hepatic artery. It runs across the triagle formed by the liver, common hepatic duct and cystic duct (Calot's triangle), to reach the gallbladder. Variations in the origin of the artery are common. It may arise from the main trunk of the hepatic artery, from the left branch of that vessel or from the gastroduodenal artery or superior mesenteric arteries. In these cases it crosses anterior (or less commonly posterior) to the common bile duct or common hepatic duct to reach the gallbladder ${ }^{3,4}$. Appreciation of the variations in ductal and arterial anatomy as they relate to the triangle are of considerable importance during excision of the gallbladder in order to avoid mistakenly ligating the common hepatic or common bile duct ${ }^{3}$. Preoperative knowledge of normal and variant arterial anatomy can lead to measures to preserve the vessels and avoid fatal injury ${ }^{5}$. The arterial patterns are of important in planning and performance of all surgical and radiological procedures in the upper abdomen. An intact hepatic artery is the gateway to successful hepatobiliary surgery. Introduction of laparoscopic cholecystectomy has stimulated a renewed interest in the anatomy of the hepatic artery. This anatomy will become even more important as computerized surgery develops ${ }^{6}$.

\section{Methods}

The present study was performed on 60 post mortem human livers collected from Bangladeshi cadavers of both sex (male - 40, female - 20), age ranging from 1 day to 75 years.

J Bangladesh Soc Physiol. 2008 Dec;(3):66-70
To collect the livers, the selected dead body was placed on the examination table in supine position and then abdominal cavity was routinely exposed by giving classical midline incision from chin to symphysis pubis. Then anterior abdominal wall retracted laterally.

Samples were collected as block dissection of liver along with associated structures like hepatic artery, portal vein, hepatic duct, part of abdominal aorta including celiac trunk and superior mesenteric artery, part of inferior venacava and other related structures like part of diaphragm, oesophagus, stomach, duodenum and pancreas. Liver of decomposed body, grossly injured livers and livers of cadavers who had undergone abdominal surgery were excluded.

All the collected specimens were from medico legal cases. Each specimen was then duly tagged by a piece of waxed cloth which bore an identifying number and then fixed in $10 \%$ formol - saline solution. All the specimens were studied by careful gross and fine dissection to observe the origin of cystic artery. Besides the typical site of origin (from right hepatic artery) this artery was also found to arise from left hepatic artery, hepatic artery proper, gastroduodenal artery and junction between left and right hepatic artery.

These findings were noted from specimen of each cadaver and collected data were expressed as percentage.

\section{Results}

In the present study, 60 human livers were selected for observation of origin of the cystic artery. Out of these livers, 54 arises from the right hepatic artery (90\%), 2 arises from the left hepatic artery (3\%), 2 arises from the junction between right and left hepatic (3\%), 1 arise from hepatic artery proper (2\%) 1 arise from gastroduodenal artery (2\%). Findings are shown in Table I and Figure 1, 2, 3 and 4. 


\section{Article}

Cystic Artery Origin

Table I: Different Origin of The Cystic artery

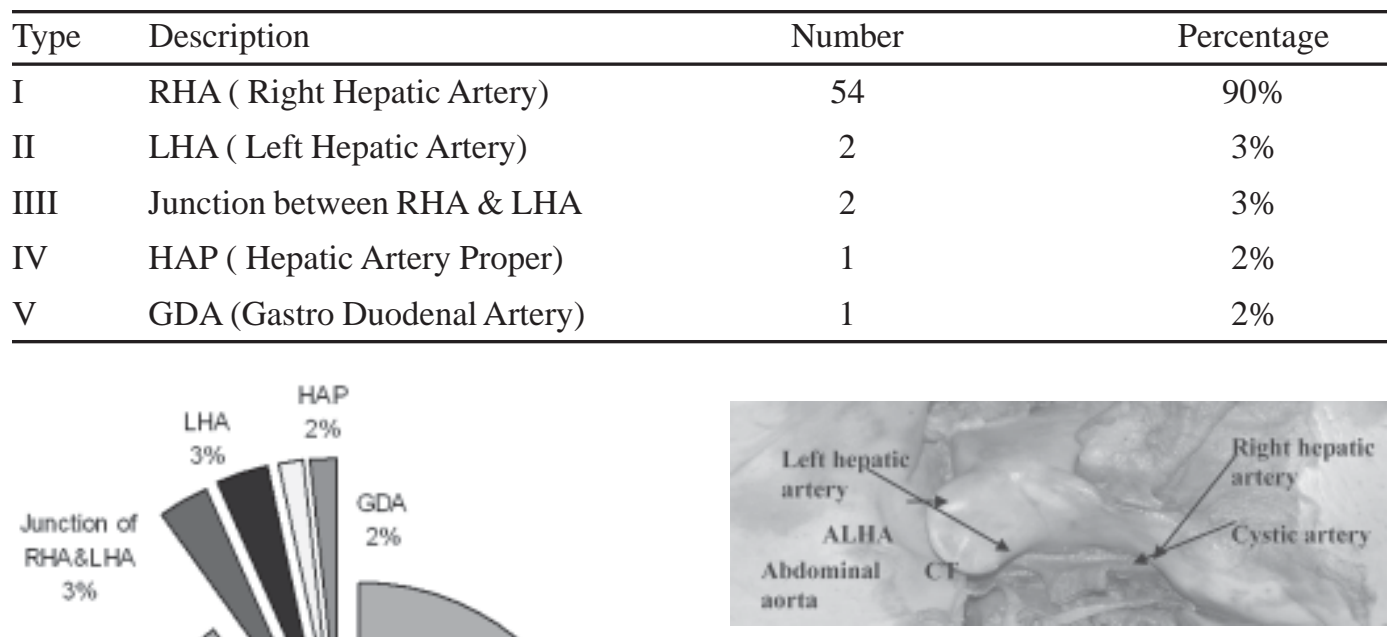

Figure 3: Photograph of a liver showing origin of the cystic artery from the left hepatic artery and origin of the accessory left hepatic artery (ALHA) from celiac trunk (CT)

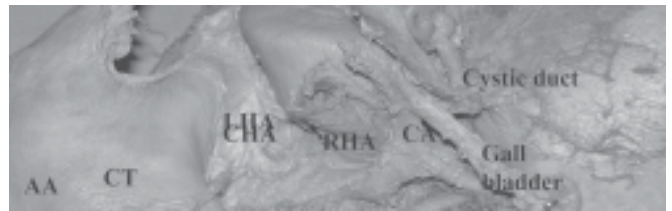

Figure 4: Photograph of the visceral surface of a liver, showing cystic artery (CA) arising from the right hepatic artery (RHA). Here $A A=$ Abdominal aorta, $C T=$ celiac trunk, $L H A=L e f t$ hepatic artery, CHA = Common hepatic artery

artery, $L H A=$ left hepatic artery, HAP = hepatic artery proper and GDA = gastroduodenal artery

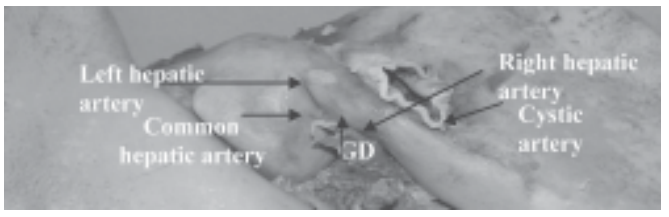

Figure 2: Photograph of the visceral surface a liver, showing the cystic artery arising from the gastroduodenal (GD) artery

\section{Discussion}

In the present study, 60 human livers were selected for observation of origin of the cystic artery. Out of these livers, 54 (90\%) took origin 68

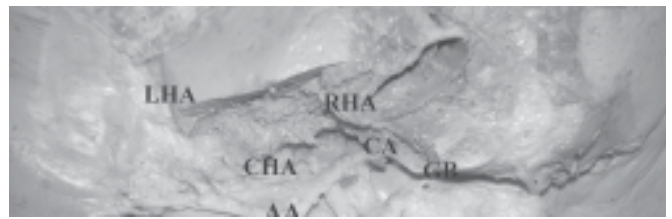

Figure 5: Photograph of the visceral surface of a liver, showing Cystic artery (CA) arising from junction between right (RHA) and left hepatic artery (LHA). Here $A A=a b d o m i n a l$ aorta, $C H A=$ Common hepatic artery, $G B=$ Gall bladder.

from the right hepatic artery and 6 (10\%) from other sources that was 2 from the left hepatic artery (3\%), 2 from the junction between right and left hepatic (3\%), 1 from hepatic artery

J Bangladesh Soc Physiol. 2008 Dec;(3):66-70 
proper (2\%) and 1 from gastroduodenal artery (2\%).

Most of the text books described that commonly the cystic artery arise from right hepatic artery and also stated that the cystic artery other than normal site of origin may arise from left hepatic or gastroduodenal or common hepatic artery $3,4,7,8,9,10,11$ which was similar to the findings of present study.

In $68 \%$ cases cystic artery was found to arise from the right hepatic artery in the BUDDE'S triangle between the hepatic duct and cystic duct, in 20\% cases all aberrant right hepatic arteries from the superior mesenteric artery give rise to the sole or second cystic artery. In $12 \%$ of cases a cystic artery was found to arise from other source (from the left hepatic or gastroduodenal artery) ${ }^{12}$. The result of the present study was nearly similar to above mentioned study.

Out of 20 caucasian cadavers (15 males and 5 females) it was found that in 15 cases cystic artery was single and arising from the right hepatic artery (75\%), in 3 cases cystic artery was found to arise from hepatic artery proper (15\%) and in 1case from left hepatic artery (5\%), whereas one cadaver exhibited two cystic arteries, each arising from the normal right hepatic artery and accessory common hepatic artery respectively ${ }^{5}$. In present study percentage of origin of cystic artery from typical site that is from right hepatic artery was more and percentage of origin of cystic artery other than right hepatic artery was less than the result of above mentioned author but site of origin was similar to present study. Approximately in $70 \%$ of cases a singular cystic artery originates from the right hepatic artery in the upper portion of the hepatobiliary triangle. When superficial and deep branches of the cystic artery do not share a common origin, it is defined as a double cystic artery occurring with a frequency of 15\%, cystic artery arise from left hepatic artery is found in roughly $1 \%$ of cases. It may arise from gastroduodenal artery (low - lying

J Bangladesh Soc Physiol. 2008 Dec;(3):66-70 cystic artery) passes inferior to the cystic duct, and therefore remains outside the hepatobilliary triangle ${ }^{13}$.

Lipshutz studied on 83 cadavers and found that cystic artery most frequently was a branch of the right hepatic artery. In 2\% of cases the cystic artery arose as a branch of the left hepatic artery and also found three times the cystic artery as branch of the gastroduodenal artery. In $8 \%$ of the cases the cystic artery is represented by two parallel vessels which arise separately from the right hepatic artery except in 2 of these subjects in which they arise from a hepatic branch of the superior mesenteric artery ${ }^{14}$. Here different site of origin of cystic artery was nearly similar to the present study but percentage of origin of cystic artery was not clearly mentioned.

Unusual anatomy of the right hepatic artery can itself affect the path and form of the cystic artery, with the most frequent variation resulting from an aberrant origin of the right hepatic artery. Generally this anomalous source is the superior mesenteric artery or more rarely the abdominal aorta. It is expected that the findings of the present study will enrich the information pool regarding various origin and location of cystic artery in Bangladeshi people. To establish a standard we need further studies adopting better techniques on larger samples from different parts of Bangladesh.

\section{Author Affiliations}

1. *Professor Dr Mohsin Khalil, MBBS, M Phil, Professor and Head of Anatomy, MMC, Mymensingh, Bangladesh

2. Dr Zinat Rezina Sultana, Department of Anatomy, MMC, Mymensingh - 2206 , Bangladesh

3. Dr Habibur Rahman Rahman, Assistant Professor of Anatomy, MMC, Mymensingh - 2206 , Bangladesh

4. Dr Seheli Zannat Sultana, Assistant Professor of Anatomy, MMC, Mymensingh - 2206 , Bangladesh

5. Dr Sabina Mannan, Assistant Professor of Anatomy, MMC, Mymensingh - 2206, Bangladesh

6. Dr Md Mahbabur Rahman, Curator of Anatomy, MMC, Mymensingh - 2206 , Bangladesh

7. Dr M Shibbir Ahamed, Lecturer of Anatomy, MMC, Mymensingh - 2206 , Bangladesh

8. Dr Md Ashraful Islam Chowdhury, Department of Anatomy, MMC, Mymensingh - 2206 , Bangladesh

* For Correspondence 


\section{Article}

Cystic Artery Origin

\section{References}

1. Moore KL, Dalley II AF. Clinically Oriented Anatomy. 4th ed. Philadelphia: Lippincott Williams \& Wilkins; 1999. pp. 221- 5, 263 - 76

2. Sarin YK. Biliary ductal and vascular anomalies associated with choledochal cyst. Journal of Indian Association of Pediatric Surgeons 2005; 10(2): 86-8.

3. Borley N.R, editor. Hepatobiliary system In: Standring S, Ellis H, Healy J C, Johnson D, Williams A, Collins P, Wigley C, editors. Gray's anatomy: the anatomical basis of clinical practice. 39th ed. Edinburgh: Elsevier Churchill Livingstone; 2005. p.1213- 29.

4. Sinnatamby CS. Last's Anatomy: regional and applied. 10th ed. Edinburgh: Churchill Livingstone; 1999. p. $253-60$.

5. Muhammad S and Ahmad RA. Duplication of hepatic artery. Saudi J Gastroenterol 2001; 7 (3): 103 - 8.

6. Jones RM, Hardy KJ. The Hepatic Artery: a reminder of surgical anatomy. J.R.Coll.Surg.Edinb., 2001; 46: 168-70.

7. Basmajian JV, Slonecker CE, editors. Grants method of Anatomy. 11th ed. Baltimore: Williams and Wilkins; 1989. p.158-69.

8. Romanes GJ. Cunningham’s Manual of Practical Anatomy (Vol. I). 15th ed. London: Oxford University Press; 1986. p. $127-8$.

9. Das S. A concise - Text book of Surgery. 1st ed. Calcutta: 1994. pp. $834-6,860-1$.

10. Garden OJ and Bismuth H. Anatomy of the liver. In Carter SD, Russell RCG, Pitt HA, and Bismath H editors. Robs and Smith's Operative Surgery. 5th ed. London: Chapman \& Hall Medicine; 1996. p. 1-4.

11. Townsend CM, Beauchamp RD, Evers BM, Mattox KL. Sabiston text book of Surgery.Volume:2. 17th ed. Elsevier: Saunders; 2004. p. 1514 - 23.

12. Vandamme JPJ, Bonte J and Schueren JVD. A revaluation of hepatic and cystic arteries. The importance of the aberrant hepatic branches. Acta anat. 1969; 73: 192-209.

13. The cystic artery branches from the hepatic artery proper 2007. Retrieved 27/01/08 from "http:// en.wikipedia.org/wiki/cystic_artery"

14. Lipshutz B. A composite study of the celiac axis artery. Anal of surgery 1917; 65: 159 - 67. 